\title{
Respuesta en Campo de Clones de Café a la Inoculación con Consorcios de Hongos Micorrízicos Arbusculares en la Región Amazonas, Perú
}

\author{
Geomar Vallejos-Torres ${ }^{(1)^{*}}$, Luis Arévalo(1), Ingrid Iliquin ${ }^{(2)}$ y Reynaldo Solis ${ }^{(1)}$ \\ (1) Instituto de Investigaciones de la Amazonía Peruana, Jr. Belén Torres de Tello 135, San Martín, Perú (e-mail: \\ gvallejost@gmail.com; larevalol@iiap.gob.pe; reynaldosolisleyva@gmail.com) \\ (2) Universidad Nacional Toribio Rodríguez de Mendoza, Calle Higos Urco 342-350-356, Chachapoyas, Amazonas, \\ Perú (e-mail: milagritosk3d09@gmail.com) \\ * Autor a quien debe ser dirigida la correspondencia
}

Recibido Ene. 3, 2019; Aceptado Mar. 13, 2019; Versión final May. 12, 2019, Publicado Dic. 2019

\begin{abstract}
Resumen
Se evalúan las respuestas en campo de clones de café a la inoculación con consorcios de hongos micorrízicos arbusculares (HMA) y la aplicación de diferentes dosis de compost en clones de café (variedad Caturra) en condiciones de campo. Los clones de café micorrizados y aclimatados fueron instalados en campo definitivo considerando un diseño en bloque completamente al azar (DBCA) con arreglo factorial: factor A (3 inóculos de HMA y un testigo sin inóculo) y factor B (2 dosis de compost y un tratamiento control sin compost), con 3 bloques. Se evaluó, 295 días después de la siembra, la altura de plantas, número de ramas, longitud de micelio extraradical, colonización micorrízica y número de esporas. Los resultados indican diferencias estadísticas significativas en todos los consorcios de HMA y dosis de compost evaluados. Por lo tanto, el estudio muestra que la inoculación de plantas clonales de café con consorcios AMF y la aplicación de compost tiene efectos positivos en el desarrollo morfológico de las plantas de café.
\end{abstract}

\section{Field response of Coffee Clones to Inoculation with Consortium of Arbuscular Mycorrhizal Fungi in the Amazonas Region, Peru}

\begin{abstract}
The field responses of coffee clones to inoculation with consortium of arbuscular mycorrhizal fungi (AMF) and the application of different doses of compost in coffee clones (Caturra variety) under field conditions are evaluated. The mycorrhized and acclimated coffee clones were installed in a definitive field considering a randomized complete block design (RCBD) with factorial arrangement: factor $\mathrm{A}$ ( 3 consortium of $\mathrm{AMF}$ and a control without inoculum) and factor B (2 doses and a control treatment without compost), with 3 blocks. After 295 days after sowing the following variables were evaluated: plant height, number of branches, length of extraradical mycelium, mycorrhizal colonization and number of spores. The results indicate significant statistical differences in all consortium of AMF and compost doses studied. Therefore, the study shows that the inoculation of coffee clonal plants with AMF consortiums and the application of compost has positive effects on the morphological development of coffee plants.
\end{abstract}

Keywords: arbuscular mycorrhizal fungi; compost; extraradical mycelium; mycorrhizal colonization. 


\section{INTRODUCCIÓN}

El café (Coffea arabica L.) tiene una importancia económica mundial, se cultiva en más de 70 países y representa uno de los productos más comercializados en el mundo, superado solo por el petróleo (Andrade et al., 2009), mientras que en el Perú es el principal producto agrícola de exportación y representa una fuente de ingresos para aproximadamente 223 mil familias (MINAGRI, 2018). El café se cultiva en 11 regiones (Del Águila et al., 2018) y Amazonas es la cuarta región cafetalera más importante del Perú (Torres y Siche, 2016). Los hongos micorrízicos arbusculares (HMA) constituyen un grupo funcional clave de la biota del suelo que contribuyen en el mejoramiento de la estructura de los suelos, la multifuncionalidad del ecosistema y la productividad de los cultivos (Mahdhi et al., 2017), además también pueden incidir positivamente en la seguridad alimentaria, al aumentar el rendimiento de importantes cultivos básicos (Rodríguez y Sanders, 2015). La caficultura peruana a pesar de su importancia económica, enfrenta limitaciones en el manejo del cultivo (deficientes prácticas culturales, agotamiento de suelos, plagas y enfermedades, etc.) que causan la disminución del rendimiento, y en este contexto, los HMA pueden ser contemplados como una alternativa de manejo sostenible del suelo.

En Latinoamérica, entre el 2008 y 2013 la producción de café fue seriamente afectado por la roya amarilla debido a factores como la situación económica, el manejo agronómico, las condiciones climáticas, etc. (Avelino et al., 2015), y una comunidad de HMA diversa y saludable en las plantaciones de café puede ayudar a amortiguar los impactos negativos de estos eventos en el cultivo (De Beenhouwer et al., 2015). En este contexto, el uso de las micorrizas representa una alternativa para el manejo sostenible del cultivo, por lo que los HMA están siendo estudiados en diversos países de América Latina y en el café, los trabajos se han centrado principalmente en evaluar la diversidad y abundancia de los HMA en diferentes sistemas de producción y el efecto de los HMA en la fisiología y mejoramiento del rendimiento (Trejo et al., 2011; Júnior et al., 2019). La propagación vegetativa permite que los clones sean genéticamente idénticos a los parentales (Solis et al., 2017) y que tengan alta calidad fitosanitaria. El desarrollo de un método efectivo de propagación vegetativa favorece la implementación de nuevas plantaciones comerciales con semilla vegetativa de alta calidad genética y fitosanitaria, y en la Amazonía Peruana se han desarrollado tecnologías de propagación vegetativa del café mediante el enraizamiento de estacas en microtúneles (Ruiz, 2015) a partir de plantas madres con alto rendimiento y tolerantes a la roya amarilla (Hemileia vastatrix), principal problema fitosanitario del café en la Amazonía Peruana, pero aún no se ha evaluado la respuesta de estos clones a la inoculación con HMA.

La información sobre las asociaciones de café con micorrizas es escasa a pesar de la gran importancia económica de este cultivo para muchos países tropicales en desarrollo (Andrade et al., 2009). En Latinoamérica ya se han realizado estudios sobre la diversidad de especies en consorcios los HMA y los efectos de estos sobre plantas de café (Osorio et al., 2002), pero en el Perú estos estudios se iniciaron en años recientes y existe muy poca información al respecto (Del Águila et al., 2018). Por lo tanto, el presente estudio tiene como objetivo evaluar las respuestas en campo de clones de café a la inoculación con consorcios de HMA y la aplicación de diferentes dosis de compost en clones de café (variedad Caturra) en condiciones de campo.

\section{METODOLOGÍA}

La presente investigación es de tipo experimental y la metodología se detalla en varias sub-secciones, donde se explica el desarrollo del ensayo y las variables de estudio evaluadas. Para el desarrollo de este estudio, los propietarios de cada una de las plantaciones de café autorizaron la colecta de muestras de suelo rizosférico para la obtención de los inóculos de HMA y la instalación de la parcela experimental.

\section{Área de estudio}

El ensayo se instaló en el centro poblado Miraflores, distrito de Huambo, provincia de Rodríguez de Mendoza, región Amazonas. Geográficamente la parcela experimental se encuentra ubicado a $06^{\circ} 26.202$ " de latitud sur y $77^{\circ} 31.467^{\prime \prime}$ de longitud oeste, a $1675 \mathrm{msnm}$. Miraflores es una de las principales comunidades productoras de café en la región Amazonas y presenta buenas condiciones edafoclimáticas para el desarrollo del cultivo, con una temperatura media anual de $19.2^{\circ} \mathrm{C}$ y una precipitación de $876 \mathrm{~mm}$ al año.

\section{Propagación vegetativa del café}

En el área de estudio se identificaron plantas de café (variedad Caturra) que presentaban alto rendimiento y tolerancia a la roya amarilla y luego fueron clonados. El enraizamiento de estacas de café en microtúneles se realizó en las en las instalaciones del Instituto de Desarrollo de la Universidad Nacional Toribio 
Rodríguez de Mendoza, ubicado en Huambo, empleando estacas de $4 \mathrm{~cm}$ de longitud con $50 \%$ de área foliar, 6 nebulizaciones por un minuto al día, 2000 ppm de ácido indolbutírico como inductor de enraizamiento y pellets Jiffy como sustrato (Ruiz, 2015). Después de un periodo de inducción de enraizamiento de 50 días las estacas enraizadas fueron transferidas a bolsas de vivero $(5 \times 8 \mathrm{~cm})$, los cuales contenían arena de río y tierra agrícola estériles en proporción 1:2 respectivamente, para el proceso de aclimatación. Las plántulas de café clonadas fueron colocadas en un vivero con sombra al $80 \%$ por un periodo de 15 días y después fueron transferidos a otro vivero que tenía sombra al $50 \%$ por 15 días más. Finalmente las plántulas fueron expuestas a la luz del sol y después de 3 meses de iniciado el proceso de aclimatación las plántulas fueron transferidas a campo para iniciar las evaluaciones. En este proceso se hizo un monitoreo diario del requerimiento hídrico y del control fitosanitario de las plantas.

\section{Colecta de inóculos de hongos micorrízicos arbusculares}

La colecta de inóculos se realizó a partir de muestras de suelo rizosférico ubicadas alrededor de las plantas de café seleccionadas, considerando un radio de $30 \mathrm{~cm}$ del tallo de la planta de café y una profundidad de $20 \mathrm{~cm}$. Las fuentes de inóculos fueron multiplicados en camas de $0.5 \mathrm{~m}^{2}$ con sustrato estéril y utilizando plantas trampas de maíz (Zea mays), por un periodo de 60 días (Del Águila et al., 2018). Las esporas fueron extraídas del suelo con base en la metodología propuesta por Gerdemann y Nicolson (1963) y cuantificadas e identificadas a nivel de morfoespecie con base a las descripciones del INVAM (2017).

\section{Inoculación de plántulas de café con hongos micorrízicos arbusculares}

La inoculación de esporas de HMA en los clones de café se realizó al mismo tiempo que la transferencia de las estacas enraizadas a las bolsas con el sustrato estéril para su aclimatación. Haciendo uso de guantes quirúrgicos y materiales esterilizados que fueron cambiados en cada tratamiento, se inocularon 3000 esporas del correspondiente consorcio de micorrizas a cada clon, colocando el inóculo cuidadosamente alrededor del hoyo en el que se colocaron las estacas enraizadas, con el fin de poner en contacto directo los HMA con las raíces de las plántulas clonales de café (Del Águila et al., 2018).

En el estudio se utilizaron 3 inóculos de HMA colectados de plantas de café tolerantes a la roya amarilla (Tabla 1) y que al ser inoculados en clones de café en condiciones de invernadero produjeron plantas con excelentes características morfológicas (Sánchez, 2017), además se utilizó un tratamiento testigo negativo (sin inóculo). Adicionalmente, al inóculo de HMA se le añadió compost, debido a que las enmiendas orgánicas contienen nutrientes que optimizan el efecto de los HMA (Tabla 2). Se utilizó compost porque es la enmienda orgánica más utilizada en el área de estudio.

Tabla 1: Procedencia de los consorcios y especies de HMA identificados en la rizósfera de plantaciones de café (Coffea arábica variedad Caturra) de tres localidades de la región Amazonas, Perú.

\begin{tabular}{|l|l|}
\hline \multicolumn{1}{|c|}{$\begin{array}{c}\text { Localidad de procedencia del } \\
\text { consorcio de HMA }\end{array}$} & \multicolumn{1}{|c|}{ Especies identificadas } \\
\hline Área de estudio & $\begin{array}{l}\text { Acaulospora foveata, Acaulospora mellea, Acaulospora sp., Glomus } \\
\text { geosporum y Glomus sp1. }\end{array}$ \\
\hline Esperanza 1 & $\begin{array}{l}\text { Acaulospora scrobiculata, Acaulospora rhemii, Acaulospora sp., } \\
\text { Glomus aggregatum y Glomus sp2. }\end{array}$ \\
\hline Omia 10 & $\begin{array}{l}\text { Acaulospora rugosa, Acaulospora foveata, Acaulospora mellea, } \\
\text { Glomus geosporum, Glomus sinuosum, Glomus sp1., Glomus sp2. y } \\
\text { Ambispora appendicula. }\end{array}$ \\
\hline Shiwa 11 & $\begin{array}{l}\text { Acaulospora tuberculata, Acaulospora rhemii, Acaulospora foveata, } \\
\text { Acaulospora sp., Glomus geosporum y Glomus sp2. }\end{array}$ \\
\hline
\end{tabular}

Tabla 2: Características fisicoquímicas del compost utilizado en este estudio.

\begin{tabular}{|l|c|c|c|c|c|c|c|c|c|c|c|c|}
\hline Muestra & $p H$ & $\begin{array}{c}M . O . \\
(\%)\end{array}$ & $\begin{array}{c}N \\
(\%)\end{array}$ & $\begin{array}{c}P \\
(p p m)\end{array}$ & $\begin{array}{c}K \\
(p p m)\end{array}$ & $\begin{array}{c}\text { Clase } \\
\text { Textural }\end{array}$ & \multirow{2}{*}{$\mathrm{CIC}$} & \multicolumn{5}{|c|}{ Cationes Cambiables } \\
\cline { 8 - 13 } & & & $\mathrm{Ca}^{+2}$ & $\mathrm{Mg}^{+2}$ & $\mathrm{~K}^{+}$ & $\mathrm{Na}^{+}$ & $\mathrm{Al}^{+3}+\mathrm{H}^{+}$ \\
\hline Compost & 6.48 & 9.48 & 0.47 & 70.64 & 2905.5 & $\begin{array}{c}\text { Arena } \\
\text { franca }\end{array}$ & 72.00 & 41.53 & 21.76 & 5.59 & 0.84 & 0.00 \\
\hline
\end{tabular}

\section{Diseño experimental}

Las plántulas de café micorrizadas y aclimatados fueron instaladas en campo definitivo cuidando de no afectar las raíces, y considerando un diseño en bloque completamente al azar (DBCA) con arreglo factorial: factor A (3 inóculos de HMA y un tratamiento testigo sin inóculo) y factor B (2 dosis de compost y un tratamiento testigo sin compost). Los tratamientos en estudio se indican en la tabla 3. El diseño 
experimental estuvo constituido por 3 bloques y 12 tratamientos, y cada tratamiento tenía 16 unidades experimentales, con un total de 576 plántulas de café en todo el estudio. Las variables en estudio fueron evaluados 295 días después de la siembra en campo definitivo.

Tabla 3: Descripción de los tratamientos de estudio.

\begin{tabular}{|c|l|c|}
\hline Tratamientos & \multicolumn{1}{|c|}{$\begin{array}{c}\text { Fuente de inóculos de HMA } \\
\text { (Consorcios) }\end{array}$} & $\begin{array}{c}\text { Dosis de abono - compost } \\
(\mathrm{g})\end{array}$ \\
\hline T1 & Testigo in inóculo & 0 \\
\hline T2 & Testigo in inóculo & 200 \\
\hline T3 & Testigo in inóculo & 0 \\
\hline T4 & Esperanza 1 & 100 \\
\hline T5 & Esperanza 1 & 200 \\
\hline T6 & Esperanza 1 & 0 \\
\hline T7 & Omia 10 & 100 \\
\hline T8 & Omia 10 & 200 \\
\hline T9 & Omia 10 & 0 \\
\hline T10 & Shiwa 11 & 100 \\
\hline T11 & Shiwa 11 & 200 \\
\hline T12 & Shiwa 11 & \\
\hline
\end{tabular}

\section{Evaluación de la colonización micorrízica}

Se colectaron 30 raíces (secundarias y terciarias) de las plantas de café a $10 \mathrm{~cm}$ del tallo principal de la planta (tres puntos diferentes) y se depositaron en un tubo Falcon de $50 \mathrm{ml}$. Luego, estas muestras se colocaron en tubos de ensayo en una solución de alcohol al 70\% y se envolvieron con cintas parafilm para que no se volatice el alcohol y se consiga una buena conservación. Estas muestras se trasladaron al laboratorio del Instituto de Investigaciones de la Amazonía Peruana - San Martín y se colocaron en una refrigeradora a $4^{\circ} \mathrm{C}$ por un periodo de 12 horas.

La tinción de raíces se realizó de acuerdo a la metodología propuesta por Phillips y Hayman (1970) con modificaciones. Para esto las raíces se colocaron en tubos de ensayo, dentro de una solución de hidróxido de potasio $(\mathrm{KOH})$ al $10 \%$ de concentración en peso/volumen, hasta cubrir la muestra por un periodo de 24 horas. Transcurrido el tiempo, fueron colocadas en baño María a $90{ }^{\circ} \mathrm{C}$ durante 30 minutos, con la finalidad de remover el contenido citoplasmático y aclarar el tejido cortical. Posteriormente, las raíces se lavaron tres veces con agua corriente y se dejaron a temperatura ambiente en agua oxigenada durante 90 minutos, para realizar el aclarado de los pigmentos de la raíz. Finalizado los 90 minutos, las raíces se lavaron con vinagre blanco para acidificar las muestras y prepararlas para la tinción. Después las raíces fueron sumergidas en tinta azul de trypano al $0.25 \%$ en concentración peso/volumen y luego se colocaron en baño María a 90 ㄷ durante 60 minutos. Las muestras de raíces se dejaron enfriar a temperatura ambiente por 10 minutos e inmediatamente se lavaron tres veces con vinagre al $50 \%$, para eliminar el exceso de la tinta. Finalmente, las raíces teñidas fueron conservadas en vinagre. La determinación del grado de colonización micorrízica fue calculada utilizando la metodología propuesta por Trouvelot et al., (1986). Para esto, en una lámina portaobjetos se colocaron verticalmente, una a continuación de la otra, los 30 fragmentos de raíz teñidas de $1 \mathrm{~cm}$. Luego se agregó una gota de lactoglicerol cada 10 raíces a fin de mantenerlas húmedas y se cubrieron con una laminilla cubreobjetos. Posteriormente, las raíces montadas se observaron en un microscopio a un aumento de 10X. La evaluación se realizó a cada segmento de raíz, asignándole un porcentaje correspondiente a la colonización micorrízica de acuerdo a las categorías indicadas en la Figura 1. Finalmente, para el cálculo de la intensidad de la colonización en el sistema radicular se utilizó la ecuación 1:

$\% \mathrm{CM}=(\mathrm{n} 1+5(\mathrm{n} 2)+30(\mathrm{n} 3)+70(\mathrm{n} 4)+95(\mathrm{n} 5)) / \mathrm{N}$

Donde:

n1 = número de fragmentos clasificados como 1

n2 = número de fragmentos clasificados como 2

n3 = número de fragmentos clasificados como 3

n4 = número de fragmentos clasificados como 4

n5 = número de fragmentos clasificados como 5 


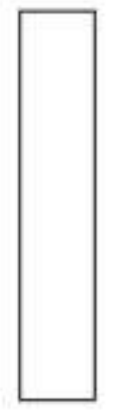

0 $0 \%$

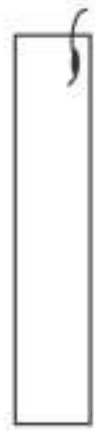

1

$<1 \%$

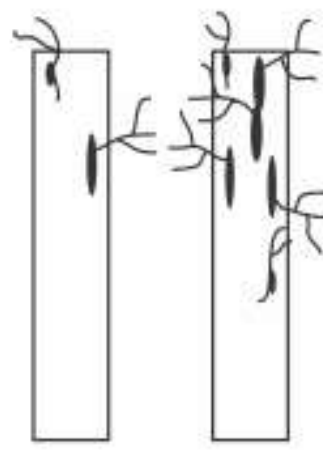

2

$<10 \%$

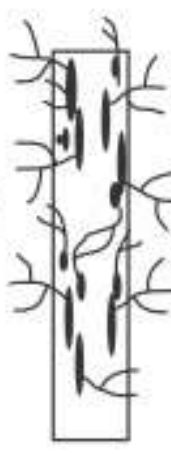

4

$>50 \%$

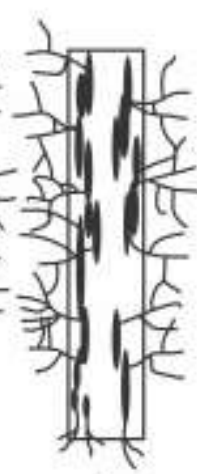

5

$>95 \%$

Figura 1: Categorías de colonización micorrízica (Trouvelot et al., 1986).

\section{Estimación de la longitud de micelio extraradical}

Para la determinación de la longitud del micelio extraradical se colectaron $10 \mathrm{~g}$ de suelo, de tres puntos diferentes, a una distancia de $30 \mathrm{~cm}$ del tallo principal de cada planta de café. Las muestras de suelo se recogieron a una profundidad de $15 \mathrm{~cm}$ y se introdujeron en bolsas Ziploc con cierre hermético. En el laboratorio, las muestras de suelo se colocaron en papel bond A4 y se secaron a temperatura ambiente. La tinción del MER se desarrolló mediante la técnica del gel semisólido y fue cuantificado por el método de intersección de cuadrantes (Carballar, 2009) con el siguiente procedimiento:

En una balanza analítica se pesó un $1 \mathrm{~g}$ de suelo seco y se colocó dentro de un vaso precipitado de $250 \mathrm{ml}$, en el cual se agregó $5 \mathrm{ml}$ de vinagre blanco para dispersar los agregados del suelo y acidificar los segmentos de micelios. Luego se añadió $20 \mathrm{ml}$ de solución de tinta "Artesco" al 15\% disuelto en ácido acético y se dejó reposar por 30 minutos a temperatura ambiente. Transcurrido los 30 minutos, se agregó agua destilada al vaso precipitado hasta completar los $100 \mathrm{ml}$. Posteriormente las muestras de suelo fueron colocadas en baño María a $90{ }^{\circ} \mathrm{C}$ por un periodo de 90 minutos, siendo esta agitada cada 30 minutos. Cumplido los 90 minutos las muestras de suelo tratadas fueron pasadas por un tamiz de malla de $38 \mu \mathrm{m}$. El material restante en el tamiz se depositó en el mismo vaso precipitado y se agregó $30 \mathrm{ml}$ de agua destilada. A continuación, estas muestras de suelo se colocaron por segunda oportunidad en baño María $\left(90{ }^{\circ} \mathrm{C}\right)$ por un minuto y se agregaron $70 \mathrm{ml}$ de agar-agar al $0.64 \%$ en concentración peso/volumen, lo cual se dejó reposar por 5 minutos, logrando una concentración final de $0.45 \%$ de la solución. Finalizado los cinco minutos, las muestras se agitaron con una jeringa de $20 \mathrm{ml}$ con el fin de mezclarlo completamente. Finalmente se tomaron $10 \mathrm{ml}$ de la solución y se colocó en una placa Petri, la cual previamente estaba cubierta en la base por una rejilla de papel cuadriculada de $0.5 \mathrm{~cm}^{2}$.

Para la estimación de la longitud de micelio extraradical, las muestras dispuestas en las placas Petri fueron observadas en un estéreo microscopio a 4.5X y con ayuda de un contómetro se cuantificó las intersecciones Hifa-Línea. El conteo de micelio se realizó en toda el área de la placa Petri haciendo un desplazamiento vertical y en recorrido ordenado, cuidando que todas las intersecciones de las líneas tanto verticales como horizontales sean contabilizadas. La cantidad numérica obtenida se trasformó a longitud de micelio por unidad de peso de suelo utilizando la ecuación 2, fórmula propuesta por Newman (1966).

$$
R=\frac{\pi A N}{2 H}
$$

Donde:

$\mathrm{R}=$ Longitud de micelio por unidad de peso de suelo

$\mathrm{A}=$ Área de la placa

$\mathrm{N}=$ Número de intersecciones

$\mathrm{H}=$ Longitud total de las líneas de la placa $(\mathrm{cm})$

En este estudio también se evaluaron la cuantificación de esporas de HMA, altura de plantas y número de ramas. 


\section{Análisis de datos}

La evaluación se realizó 295 días después de la instalación de las plantas de café en condiciones de campo. Los datos fueron sometidos al análisis de varianza y las medias se compararon mediante la prueba de Tukey con una probabilidad del $5 \%$. Los datos fueron analizados empleando el software estadístico $\mathrm{R}$ versión 5.3.1., y las figuras se realizaron utilizando Microsoft Excel versión 15.0.

\section{RESULTADOS Y DISCUSIÓN}

El gobierno peruano a través del Plan Nacional de Acción del Café Peruano promueve el crecimiento económico, equitativo, competitivo y sostenible basado en las potencialidades de desarrollo de cada territorio, facilitando su articulación al mercado nacional e internacional, y asegurando el aprovechamiento sostenible y conservación de los recursos naturales (MINAGRI, 2018). En este contexto es necesario desarrollar prácticas sostenibles y tecnologías de manejo agronómico que puedan ser adoptados por los caficultores para incrementar la productividad. Los HMA mejoran la capacidad de las plantas para aprovechar los nutrientes del suelo e influyen positivamente en el desarrollo y productividad de los cultivos (Andrade et al., 2009), pero las comunidades de HMA difieren por efectos estacionales y la propia sucesión que se establece por la dinámica de esporulación entre las especies (Trejo et al., 2011). Por lo tanto, los efectos de los HMA en condiciones de monocultivo son limitados, siendo recomendable la implementación de policultivos y/o la inclusión de árboles en los agroecosistemas.

Las plantas de café que fueron inoculados con consorcios de HMA mostraron resultados beneficiosos, con mejor respuesta en altura de planta y numero de ramas y en los parámetros fúngicos como la colonización micorrízica, longitud del micelio extraradical y número de esporas de HMA (Tabla 4). Las plantas del tratamiento sin inoculación de los consorcios de HMA presentaron un buen resultado en la colonización micorrízica, pero tuvieron menor desarrollo en altura y numero de ramas en comparación a las plantas que recibieron los inóculos de HMA. La colonización micorrízica en estas plantas se debió a la diversidad de HMA pre-existente en el área en el que se instaló el experimento, pero al adicionar nuevas especies a través de inóculos colectados de plantas de café con alto rendimiento y tolerantes a la roya amarilla se incrementó la diversidad de HMA y se mejoró la respuesta en el desarrollo morfológico de las plantas inoculadas con HMA, debido a que la aplicación de micorrizas en los cultivos mejora la capacidad de absorción de nutrientes de las plantas (Gianinazzi et al., 2010). Además se observó diferencias significativas entre los consorcios de HMA utilizados en las distintas variables de evaluación, siendo Omia 10, el consorcio con mayor diversidad de especies, el que mostró mejores resultados. En el mismo sentido, Trejo et al., (2011) reportó que los diferentes consorcios de HMA que evaluaron también presentaron diferencias significativas en variables de desarrollo morfológico. Las diferencias observadas en las respuestas de los diferentes inóculos de HMA se debieron a que las diversas especies que conforman los consorcios producen efectos distintos debido a la especificidad ecológica y a la compatibilidad funcional entre los HMA y las plantas de café.

En este estudio se observó que la longitud del micelio extraradical está directamente relacionado con la longitud de las plantas de café (Tabla 4), resultado que mantienen concordancia con el reporte de Del Águila et al., (2018) quien observó una relación positiva entre la longitud del micelio extraradical con la altura de plantas y la biomasa seca de la raíz. Del mismo modo, Al-Areqi et al., (2014) reportó que las plantas de café inoculadas con HMA en su estado vegetativo inicial producen mayor biomasa radicular. Estos resultados indican que los consorcios de HMA influyen positivamente en el desarrollo morfológico de plantas de café, pero para obtener mejores resultados los consorcios deben tener mayor diversidad de especies de HMA (Trejo et al., 2011).

Tabla 4: Efectos de los consorcios de HMA en plantas de café, evaluado 295 días después de establecidos en condiciones de campo.

\begin{tabular}{|l|c|c|c|c|c|}
\hline Consorcio de HMA & $\begin{array}{c}\text { Altura de } \\
\text { planta (cm) }\end{array}$ & $\begin{array}{c}\text { Número de } \\
\text { ramas }\end{array}$ & $\begin{array}{c}\text { Colonización } \\
\text { micorrízica (\%) }\end{array}$ & $\begin{array}{c}\text { Micelio } \\
\text { extraradical (cm) }\end{array}$ & $\begin{array}{c}\text { Número de } \\
\text { esporas de HMA }\end{array}$ \\
\hline Testigo sin inóculo & $29.59 \mathrm{c}$ & $12.56 \mathrm{c}$ & $49.17 \mathrm{a}$ & $84.55 \mathrm{c}$ & $55.56 \mathrm{~b}$ \\
\hline Esperanza 1 & $34.02 \mathrm{~b}$ & $15.28 \mathrm{a}$ & $39.26 \mathrm{c}$ & $87.75 \mathrm{c}$ & $36.56 \mathrm{c}$ \\
\hline Omia 10 & $36.76 \mathrm{a}$ & $14.97 \mathrm{ab}$ & $44.01 \mathrm{~b}$ & $117.45 \mathrm{a}$ & $67.89 \mathrm{a}$ \\
\hline Shiwa 11 & $34.03 \mathrm{~b}$ & $14.53 \mathrm{~b}$ & $48.52 \mathrm{a}$ & $101.54 \mathrm{~b}$ & $71.22 \mathrm{a}$ \\
\hline
\end{tabular}

Los abonos orgánicos como el compost estimulan la actividad microbiana en el suelo y mejoran la nutrición de las plantas. Luego de analizar la respuesta de la aplicación de compost sobre el desarrollo morfológico 
de las plantas de café se observó que la dosis de $100 \mathrm{~g}$ mostró mejores resultados, pero el número de esporas es una variable que no presentó diferencias significativas en las diferentes dosis aplicadas (Tabla $5)$.

Tabla 5: Efectos de las dosis de compost en plantas de café, evaluado 295 días después de establecidos en campo.

\begin{tabular}{|c|c|c|c|c|c|}
\hline Dosis de abono $(\mathrm{g})$ & $\begin{array}{c}\text { Altura de } \\
\text { planta }(\mathrm{cm})\end{array}$ & $\begin{array}{c}\text { Número de } \\
\text { ramas }\end{array}$ & $\begin{array}{c}\text { Colonización } \\
\text { micorrízica (\%) }\end{array}$ & $\begin{array}{c}\text { Micelio } \\
\text { extraradical }(\mathrm{cm})\end{array}$ & $\begin{array}{c}\text { Número de } \\
\text { esporas de HMA }\end{array}$ \\
\hline 0 & $31.50 \mathrm{c}$ & $13.42 \mathrm{c}$ & $46.42 \mathrm{a}$ & $92.09 \mathrm{~b}$ & $56.33 \mathrm{ab}$ \\
\hline 100 & $35.99 \mathrm{a}$ & $15.73 \mathrm{a}$ & $47.52 \mathrm{a}$ & $104.96 \mathrm{a}$ & $55.33 \mathrm{~b}$ \\
\hline 200 & $33.31 \mathrm{~b}$ & $13.85 \mathrm{~b}$ & $41.78 \mathrm{~b}$ & $96.43 \mathrm{~b}$ & $61.75 \mathrm{a}$ \\
\hline
\end{tabular}

La relación simbiótica que se establece entre los HMA y las raíces de las plantas aumenta la capacidad de absorción de nutrientes disponibles en el suelo promoviendo un mayor desarrollo vegetativo de las plantas, mientras que los HMA se benefician con el suministro de fuentes carbonadas provenientes de la planta (Martín et al., 2010). En la interacción entre los consorcios de HMA y las dosis de compost aplicadas a las plantas de café se observó que la altura de planta fue mayor en el consorcio de HMA Omia 10 y la aplicación de $100 \mathrm{~g}$ de compost, mientras que el valor más bajo se obtuvo en el tratamiento testigo in inóculo de HMA y sin la aplicación de compost (Fig. 1). Del mismo modo, Trejo et al., (2011) reportó que las plantas de café inoculadas con HMA y evaluadas en condiciones de vivero presentaron mayor altura de planta en comparación con plantas control, mientras que Osorio et al., (2002) observó que el crecimiento de las plantas mejoró con la aplicación de la enmienda orgánica y se incrementó aún más con la inoculación de micorrizas, pero se redujo severamente en el suelo no modificado. La respuesta de las plantas probablemente se debe a que el café es un cultivo dependiente de la asociación micorrízica. Para poder optimizar la respuesta positiva de las plantas de café a la inoculación de los consorcios micorrízicos, Alarcón y Ferrera-Cerrato (2000) determinaron que el mejor momento para establecer la simbiosis entre los HMA y las plantas de café es el estado vegetativo inicial porque garantiza un mayor número de plantas vigorosas para el trasplante a campo definitivo, por lo tanto la inoculación micorrízica debe realizarse al momento del trasplante de las plántulas.

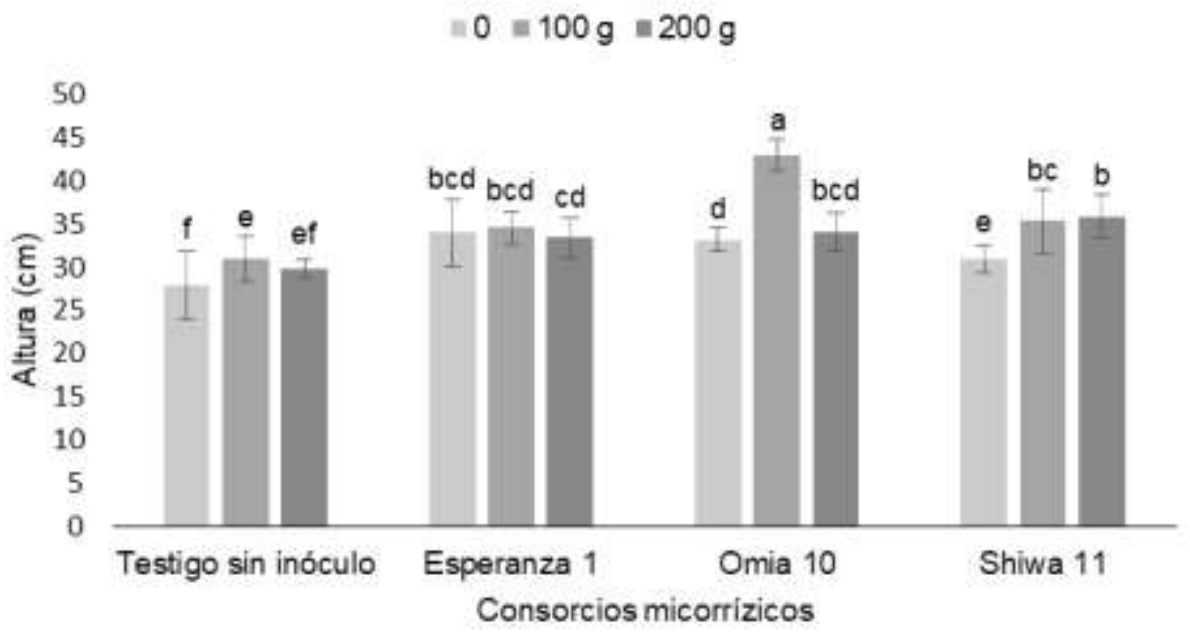

Fig. 2: Efectos de los consorcios micorrízicos y las dosis de compost en la altura de plantas de café, evaluado a los 295 días después de establecidos en campo.

Al igual que en el caso de la altura de las plantas, el valor obtenido en la variable número de ramas también fue superior cuando se empleó el consorcio Omia 10 y se aplicó $100 \mathrm{~g}$ de compost, mientras que el valor estadísticamente inferior se obtuvo en el tratamiento testigo in inóculo de HMA y sin la aplicación de compost (Fig. 3). Estos resultados concuerdan con Osorio et al., (2002) y Del Águila et al., (2018) quienes reportaron que las plantas de café inoculadas con micorrizas presentan mejores características anatómicas en comparación con plantas que no han sido inoculadas con HMA. La efectividad de la inoculación con HMA depende de la selección de cepas adecuadas, del suministro de nutrientes y de la riqueza del sustrato en el que se desarrollan las plantas debido a que los microorganismos del suelo contribuyen en el funcionamiento adecuado de los agrosistemas y ayudan en los procesos de recuperación de suelos degradados (Cruz-Hernández, 2014). En este estudio se observó que la aplicación de compost genera efectos positivos sobre el número de ramas, y esto puede haber estado influenciado por la diversidad de especies en el consorcio de HMA Omia 10, por la riqueza del sustrato y las características del suelo, 
considerando que el uso de suelo previo en el área que se instaló el experimento fue una vegetación secundaria de tres años.

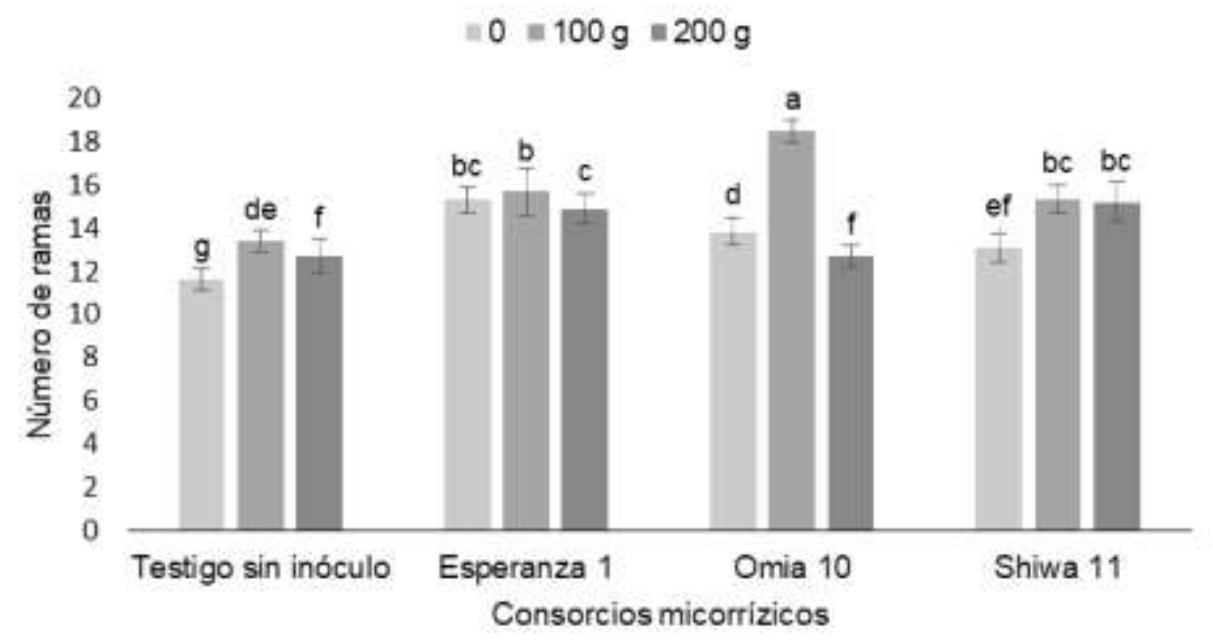

Fig. 3: Efectos de los consorcios micorrízicos y las dosis de compost en el número de ramas de plantas de café, evaluado a los 295 días después de establecidos en campo.

Los HMA colonizan primero las raíces de la planta hospedera y luego el suelo circundante, ambos a través de sus hifas (Jansa et al., 2013). La colonización micorrízica corresponde al porcentaje de ocupación de los HMA en las raíces del café, y en este estudio la colonización micorrízica obtuvo el mejor resultado en la interacción entre el tratamiento testigo que no tenía inóculo pero el suelo si presentaba diversidad de HMA propios de la zona de estudio (Fig. 4). Contrariamente, Osorio et al., (2002) reportó que las raíces de plántulas de café inoculadas fueron más altamente colonizadas por HMA que aquellas plántulas sin inocular. A su vez, Cruz-Hernández (2014) encontró que las plantas de tabaco que no habían sido sometidos a los inóculos con HMA presentaron porcentajes de colonización micorrízica que oscilan entre 22,3 y $22,7 \%$, mientras que las plantas que fueron inoculadas con HMA presentaron un máximo de $48,7 \%$ de colonización micorrízica. En este estudio los diversos consorcios de HMA presentaron entre 36,5 y $53 \%$ de colonización, pero fueron estadísticamente inferiores con respecto al tratamiento en el que no se inoculó HMA y se usó $100 \mathrm{~g}$ de compost. Este resultado puede estar relacionado con el contenido de nutrientes del suelo y la diversidad previa de HMA que influyeron directamente en la eficiencia de la micorrización de las plantas. Si bien este tratamiento sin inóculo presentó mejores resultados en la micorrización, la respuesta sobre el desarrollo vegetativo de las plantas fue menor y esto indica la importancia de enriquecer la diversidad previa de HMA existente en el suelo en el que se instalan las plantaciones con la aplicación de cepas que influyan en el desarrollo vegetativo y en la productividad de los cultivos previamente identificadas y seleccionadas.

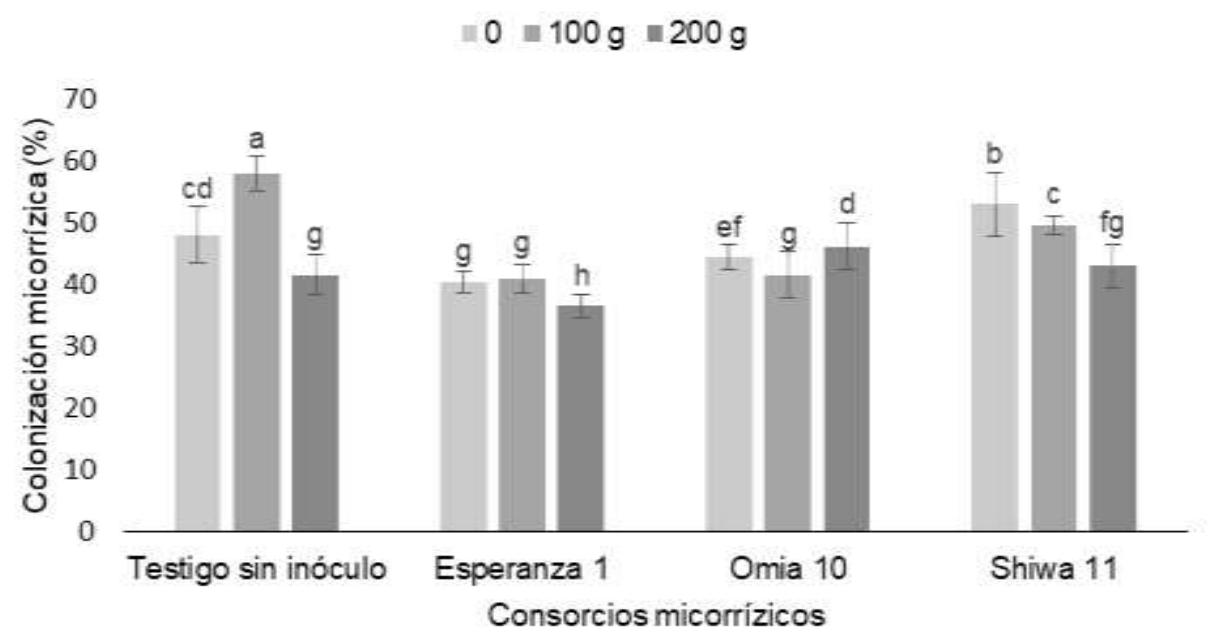

Fig. 4: Efectos de los consorcios micorrízicos y las dosis de compost en la colonización micorrízica en plantas de café, evaluado a los 295 días después de establecidos en campo. 
La longitud del micelio extraradical determina la presencia de los HMA en el suelo circundante a las raíces donde ocurre la simbiosis (Helgason y Fitter, 2009) y en este estudio, la mayor longitud del micelio extraradical se obtuvo en el tratamiento que incluía el uso del consorcio de HMA Omia 10 y la adición de $100 \mathrm{~g}$ de compost, mientras que los resultados estadísticamente inferiores se obtuvieron en el tratamiento sin inóculo y sin aplicación de compost (Fig. 5), lo que indica que los consorcios de HMA utilizados fueron capaces de establecer la simbiosis micorrízica con la plantas de café. A la misma vez, el tratamiento que incluye el uso del consorcio Omia 10 y la adición de $100 \mathrm{~g}$ de compost presentó la mayor longitud del micelio extraradical y también presentó un bajo porcentaje de colonización micorrízica con respecto a los demás tratamientos, lo que explica que esta variable no depende del grado de colonización sino de la diversidad de especies que conforman los consorcios de HMA, tal como fue reportado por Del Águila et al., (2018). Otro punto importante a resaltar es que existe una relación entre la longitud del micelio extraradical y el desarrollo morfológico de las plantas de café, confirmando lo reportado por Del Águila et al., (2018), quienes reportan que la función nutricional de las plantas se lleva a través del micelio extraradical, logrando así influir favorablemente en el crecimiento de las plantas.

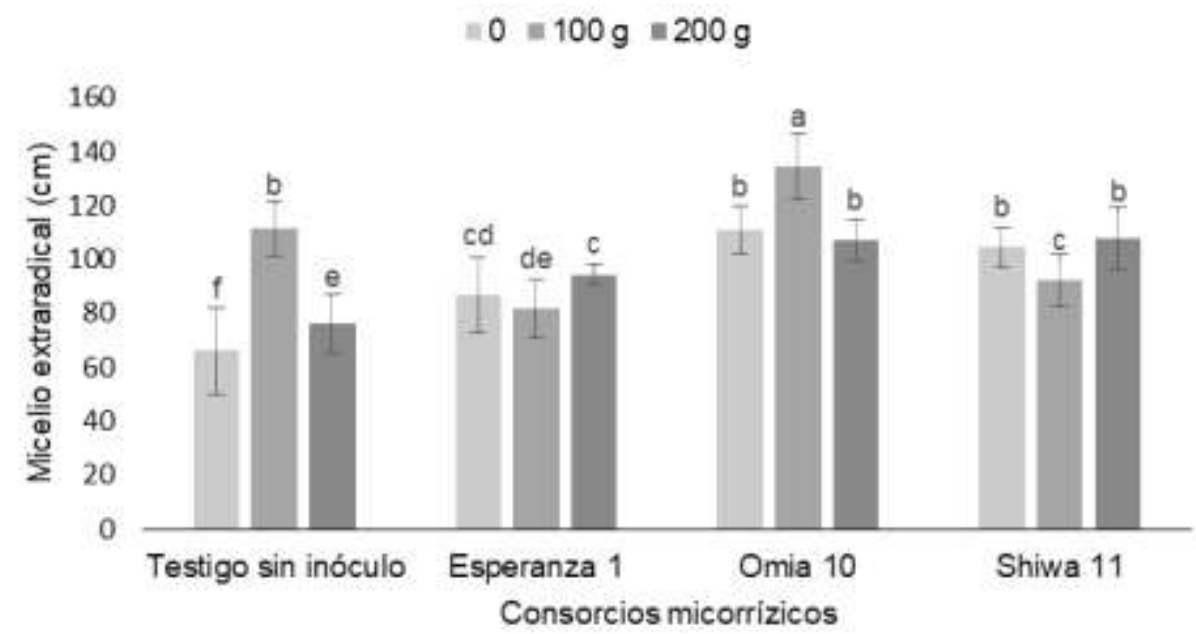

Fig. 5: Efectos de los consorcios micorrízicos y las dosis de compost en la longitud del micelio extraradical de plantas de café, evaluado a los 295 días después de establecidos en campo.

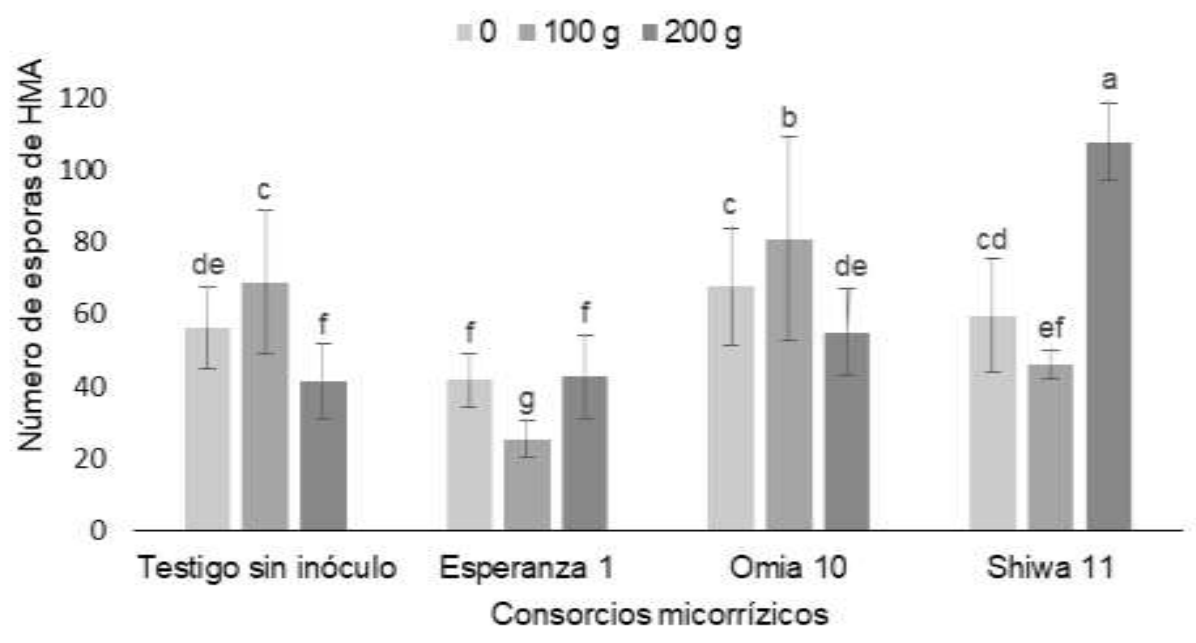

Fig. 6: Efectos de los consorcios micorrízicos y las dosis de compost en el número de esporas de HMA en plantas de café, evaluado a los 295 días después de establecidos en campo.

La variación en el número de esporas puede estar relacionado con factores que influyen en la micorrización tales como el pH, compactación de suelo, densidad aparente, porosidad y manejo agronómico, entre otros (Cardona et al., 2005). En este estudio el número de esporas presentó diferencias significativas entre los distintos consorcios, siendo Omia 10 y Shiwa 11 con aplicación de compost, los que presentaron el mayor número de esporas (Fig. 6). El pH, la humedad del suelo y la disponibilidad de nutrientes influyen en la 
colonización y en el número de esporas producidas por los HMA y en este caso el pH del suelo en el que se instaló el experimento oscilaba entre 4,3 y 4,72 favoreciendo la reproducción de los hongos.

El consorcio de HMA más efectivo en el crecimiento de las plantas fue Omia 10 y este presentó el mayor número de especies con respecto a los demás consorcios, apoyando las hipótesis que indican que los ecosistemas con tendencia a la sustentabilidad tienen mayor riqueza de especies de HMA (Trejo et al., 2011), puesto que un mayor número de especies de HMA en un consorcio, representa la suma de las posibles interacciones fúngicas con diferentes habilidades competitivas, fisiológicas e infectivas, que definen su beneficio en las plantas. En este contexto, los HMA representan alternativas sostenibles de nutrición para las plantas, que influyen positivamente en el desarrollo vegetativo y la productividad, además permiten la recuperación de suelos degradados y la sustitución parcial o total de los fertilizantes minerales.

\section{CONCLUSIONES}

El estudio demuestra que la inoculación de las plantas clonales de café con consorcios de HMA y la aplicación de compost tiene efectos positivos en el crecimiento de las plantas de café tanto para variables morfológicas (altura de planta y número de ramas) como para variables de desarrollo de los HMA (colonización micorrízica, longitud de micelio extraradical y número de esporas), propiciando así un mejor desarrollo vegetativo y plantas más vigorosas en condiciones de campo. Las mejores respuestas en el desarrollo morfológico de las plantas, el micelio extraradical y el número de esporas se obtuvieron con el consorcio Omia 10 y la aplicación de $100 \mathrm{~g}$ de compost por planta, pero una mayor colonización micorrízica se obtuvo con el tratamiento testigo sin inóculo y $100 \mathrm{~g}$ de compost.

\section{AGRADECIMIENTOS}

Los autores agradecen al Programa Nacional de Innovación para la Competitividad y Productividad INNOVATE PERÚ por financiar el presente trabajo de investigación en el marco de las actividades del proyecto "Aplicación de técnicas innovadoras en la propagación clonal e inoculación micorrízica de plantas matrices de café (Coffea arabica L.) con alta productividad en la región Amazonas", CONVENIO № 141 PNICP - PIAP - 2015.

\section{REFERENCIAS}

Al-Areqi, A.H., M. Chliyeh y otros dos autores, Effect of a composite endomycorrhizal inoculum on the growth of Coffea arabica seedlings, International Journal of Plant, Animal and Environmental Sciences, ISSN: 2231-4490, 4(1), 185-194 (2014).

Alarcón, A. y R. Ferrera-Cerrato, Manejo de la micorriza arbuscular en sistemas de propagación de plantas frutícolas, Montecillo, Estado de México, Terra, ISSN: 2395-8030, 17(3), 179-191 (2000).

Andrade, S., P. Mazzafera y otros dos autores, Arbuscular mycorrhizal association in coffee, doi: 10.1017/S0021859608008344, The Journal of Agricultural Science, 147, 105-115 (2009).

Avelino, J., M. Cristancho y otros ocho autores, The coffee rust crises in Colombia and Central America (2008-2013): impacts, plausible causes and proposed solutions, doi: 10.1007/s12571-015-0446-9, Food Security, 7(2), 303-321 (2015).

Carballar, S., Variación temporal de la diversidad de hongos de micorriza arbuscular y el potencial micorrízico en especies silvestres de Agave en Oaxaca, Tesis para optar el grado de Magister, Departamento de Conservación y Aprovechamiento de Recursos Naturales, Instituto Politécnico Nacional, Oaxaca, México, pp. 72 (2009).

Cardona, G.I., A.L. Arcos y U.G. Murcia, Abundancia de actinomicetes y micorrizas arbusculares en paisajes fragmentados de la Amazonía Colombiana, Agronomía Colombiana, ISSN: 2357-3732, 23(2), 317-326 (2005).

Cruz-Hernández, Y., M. García-Rubido y otros dos autores, Influencia de la aplicación de micorrizas arbusculares y la reducción del fertilizante mineral en plántulas de tabaco, Cultivos Tropicales, ISSN: 1819-4087, 35(1), 21-24 (2014).

De Beenhouwer, M., M. Van Geel y otros cuatro autores, Changing soil characteristics alter the arbuscular mycorrhizal fungi communities of Arabica coffee (Coffea arabica) in Ethiopia across a management intensity gradient, doi: 10.1016/j.soilbio.2015.08.037, Soil Biology and Biochemistry, 91, 133-139 (2015).

Del Águila K.M., G. Vallejos-Torres y otros dos autores, Inoculación de consorcios micorrícicos arbusculares en Coffea arabica, variedad Caturra en la región San Martín, doi: 10.4067/S0718-07642018000100137, Información Tecnológica, 29(1), 137-146 (2018).

Gerdemann, J. y T. Nicolson, Spores of mycorrhizal Endogone species extracted from soil by wet sieving and decanting, doi: 10.1016/S0007-1536(63)80079-0, Transactions of the British Mycological Society, 46, 235-244 (1963).

Gianinazzi, S., A. Gollote y otros cuatro autores, Agroecology: the key role of arbuscular mycorrhizas in ecosystem services, doi: 10.1007/s00572-010-0333-3, Mycorrhiza, 20(8), 519-530 (2010). 
Helgason, T. y A.H. Fitter, Natural selection and the evolutionary ecology of the arbuscular mycorrhizal fungi (Phylum Glomeromycota), doi: 10.1093/jxb/erp144, Journal of Experimental Botany, 60(9), 2465-2480 (2009).

INVAM, International culture collection of (vesicular) arbuscular mycorrhizal fungi, West Virginia University (2017).

Jansa, J., P. Bukovská y M. Gryndler, Mycorrhizal hyphae as ecological niche for highly specialized hypersymbionts - or just soil free-riders?, doi: 10.3389/fpls.2013.00134, Frontiers in Plant Science, 4, 134 (2013).

Júnior, P.P., B.C. Moreira y otros seis autores, Agroecological coffee management increases arbuscular mycorrhizal fungi diversity, doi: 10.1371/journal.pone.0209093, PLoS ONE, 14(1), e0209093 (2019).

Mahdhi M., T. Tounekti y otros dos autores, Composition of the root mycorrhizal community associated with Coffea arabica in Fifa Mountains (Jazan region, Saudi Arabia), doi: 10.1002/jobm.201700075, Journal of Basic Microbiology, 57, 691-698 (2017).

Martín, G.M., L. Arias y R. Rivera, Selección de las cepas de hongos micorrízicos arbusculares (HMA) más efectivas para la Canavalia ensiformis cultivada en suelo Ferralítico Rojo, Cultivos Tropicales, ISSN: 1819-4087, 31(1), 27-31 (2010).

MINAGRI, Plan nacional de acción del café peruano, Ministerio de Agricultura y Riego del Perú, pp. 53 (2018).

Newman, E.I., A method of estimating the total length of root in a sample, doi: 10.2307/2401670, Journal of Applied Ecology, 3(1), 139-145 (1966).

Osorio, N.W., J.M. Alzate y G.A. Ramirez, Coffee seedling growth as affected by mycorrhizal inoculation and organic amendment, doi: 10.1081/CSS-120004291, Communications in Soil Science and Plant Analysis, 33, 1425-1434 (2002).

Phillips, J.M., y D.S. Hayman, Improved procedures for clearing roots and staining parasitic and vesicular-arbuscular mycorrhizal fungi for rapid assessment to infection, doi: 10.1016/S0007-1536(70)80110-3, Transactions of the British Mycological Society, 55 (1), 158-161 (1970).

Rodriguez, A. y I.R. Sanders, The role of community and population ecology in applying mycorrhizal fungi for improved food security, doi: 10.1038/ismej.2014.207, The ISME Journal, 9, 1053-1061 (2015).

Ruiz, W. Propagación de café (Coffea arabica L.) mediante el enraizamiento de rebrotes utilizando cinco dosis de ácido indolbutírico y cuatro sustratos, en ambientes controlados, Tesis para optar el título de Ingeniero Agrónomo, Universidad Nacional de San Martín,Tarapoto, Perú, pp. 98 (2015).

Sánchez, T. Efecto de inóculos de hongos micorrízicos arbusculares en plantas clonales de café (Coffea arabica L.) variedad Caturra en condiciones de invernadero, Rodríguez de Mendoza, región Amazonas, Tesis para optar el título de Ingeniero Agrónomo, Universidad Nacional Toribio Rodríguez de Mendoza, Chachapoyas, Perú, pp. 78 (2017).

Solis, R., M. Pezo y otros tres autores, Vegetative propagation of Plukenetia polyadenia by cuttings: effects of leaf area and indole-3-butyric acid concentration, doi: 10.1590/1519-6984.20415, Brazilian Journal of Biology, 77(3), 580-584 (2017).

Torres, E. y R. Siche, Sostenibilidad ambiental de dos sistemas de producción de café en Perú: orgánico y convencional, Revista Ciencia y Tecnología, ISSN: 2306-2002, 12(3), 51-65 (2016).

Trejo, D., R. Ferrera-Cerrato y otros cuatro autores, Efectividad de siete consorcios nativos de hongos micorrízicos arbusculares en plantas de café en condiciones de invernadero y campo, doi: 10.4067/S0716-078X2011000100002, Revista Chilena de Historia Natural, 84, 23-31 (2011).

Trouvelot, A., J.L. Kough y V. Gianinazzi-Pearson, Mesure du taux de mycorrhization VA d'un système radiculaire. Recherche de methods d'estimation ayantune signification function elle, En: "Physiological and genetical aspects of mycorrhizae", Gianinazzi-Pearson, V. y S. Gianinazzi, Eds., INRA, Paris, 101-109 (1986). 
